\title{
The effect of similar surface features on students' understanding of the interaction of charges with electric and magnetic fields
}

\author{
Eder Hernandez, Esmeralda Campos, Pablo Barniol \\ Tecnologico de Monterrey, Monterrey, Mexico \\ Genaro Zavala \\ Escuela de Ingenieria y Ciencias, Tecnologico de Monterrey, Monterrey, Mexico \\ Facultad de Ingenieria, Universidad Andres Bello, Santiago, Chile
}

The literature in student problem-solving abilities in PER has pointed out that novices tend to focus on the surface features of a problem, instead of the underlying principles. In Electricity and Magnetism (E\&M) courses, students can confuse the concepts of electric and magnetic fields, which are often represented in problems with similar surface features. It is important to compare the students' performance on these type of problems to evaluate their understanding of these topics. With this aim, we conducted a study to explore students' performance in E\&M open-ended problems presented with similar surface features. We applied two versions of a questionnaire, one with electricity and one with magnetism problems, to 322 introductory E\&M students. In this study, we present some results focusing on a comparison between students' understanding of electric and magnetic fields in questions regarding electric charges in uniform fields, both at rest and in motion. 


\section{INTRODUCTION}

Many studies have analyzed students' understanding of electricity and magnetism concepts, e.g., [1]. The literature in student problem-solving abilities has pointed out that novices tend to focus on the surface features of a problem, instead of focusing, as an expert would, on the underlying principles [2]. This latter applies to electricity and magnetism concepts. To an expert, electricity and magnetism are very similar and closely related phenomena by their somewhat symmetric presence in Maxwell's equations and the Lorentz force equation; to a novice, electricity and magnetism may also be seen as very similar, but for their many analogous surface features [3].

The present study focuses on students' understanding of the interaction of charges with electric and magnetic fields. These are often represented in problems with similar surface features. Some studies have demonstrated that students confuse electric and magnetic fields [4]. More specifically, some research [5] has shown that students answer magnetic force questions as though they were electric force questions, thinking that the force experienced by a moving charged particle is in the direction of the magnetic field lines. Moreover, and more commonly, some other studies [3] have shown that the converse is also true: after students learn about magnetic force, they answer electric force questions as though they were magnetic force questions.
The objective of this study is to compare students' understanding of electric and magnetic fields in questions regarding electric charges in uniform fields, both at rest and in motion. We propose that the similarities of the representations may elicit the confusion between electricity and magnetism concepts. The relevance of this article is that it provides important information about students' confusion between concepts of electricity and magnetism, which helps us to have a complete vision of the students' understanding of electricity and magnetism concepts. Our contribution is to show that not only students use magnetism concepts to answer electricity questions when charges are at motion [3], but they do so as well when charges are at rest and, in some cases, they even include well-studied mechanics naïve ideas.

\section{METHODS}

We conducted this research in a large private Mexican university. The participants in this research were engineering students finishing a calculus-based course on Electricity and Magnetism (E\&M). This course is the last of three introductory physics courses offered in this institution. The course consists of three hours of lecture and 1.5 hours of laboratory session per week. Students use a known textbook [6] and Tutorials for Introductory Physics [7].

Question 1. The following figure shows a point charge $+q$ at rest in a uniform electric (or magnetic) field Eo (or Bo) with direction into the page. Draw in the figure the electric (or magnetic) force on the charge. Describe how the electric (or magnetic) force is on the charge and explain your reasoning. If there is no electric (or magnetic) force on the charge, indicate it explicitly.

Electricity

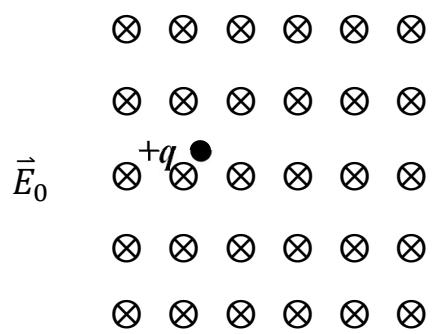

Magnetism

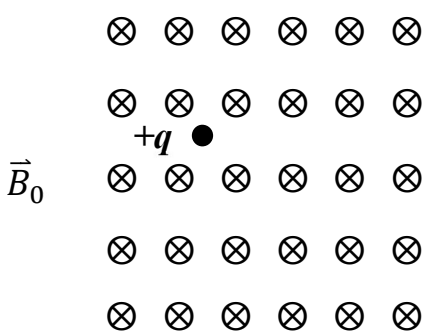

Question 2: The following figure shows a point charge $+q$ with velocity $v$ to the right in a uniform electric (or magnetic) field Eo (or Bo) with an upward direction. Draw in the figure the electric (or magnetic) force on the charge. Describe how the electric (or magnetic) force is on the charge and explain your reasoning. If there is no electric (or magnetic) force on the charge, indicate it
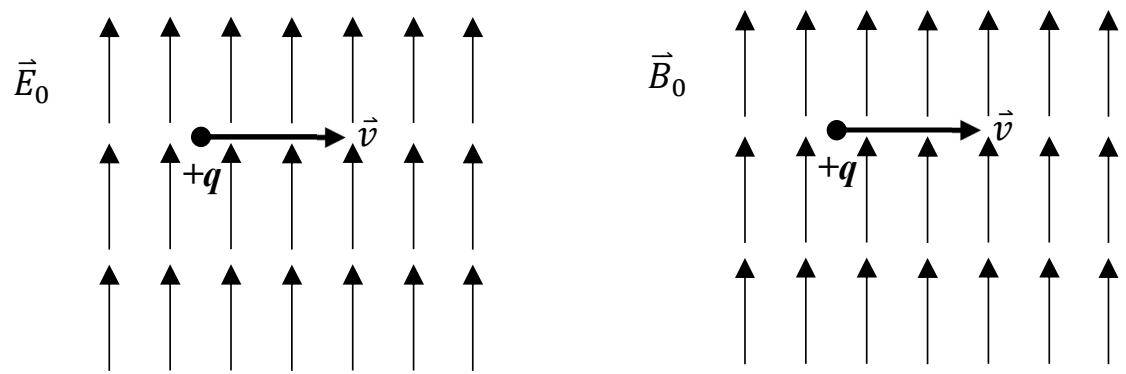

Figure 1. Set of two questions of a charge inside a uniform field. In the first question, the charge is at rest, and in the second, in motion. In the electricity version, the charge is in a uniform electric field region, and in the magnetism version, in a uniform magnetic field region. We present the test of the electricity version and include in parentheses the words that are different in the magnetism version. 
We designed two versions of an open-ended questionnaire with isomorphic surface features: one within the context of electricity and the other within the context of magnetism. Figure 1 shows the four questions used in this study. Question 1 assesses students' understanding of the force exerted by a uniform field on a charge at rest. Question 2 evaluates their understanding for a charge in motion. We presented each set of items with both electric and magnetic fields.

We administered the questionnaires to 322 introductory E\&M students during the last week of the course (after the instruction of both, electricity and magnetism topics): 160 students answered the electricity test and 162 students, the magnetism version. The selection of students to present one or the other version of the questionnaire was made randomly. The questionnaires were administered in Spanish.

We analyzed the results using a phenomenographic approach, which has been used in physics education research to analyze open-ended questions and derive students' understanding and difficulties when learning physical concepts [8]. We created the first categories based on the answers from 20 students, reaching consensus between experts. We then analyzed the answers from all the students between two of the authors, applying the emerging categories. If more categories emerged, we included them in the analysis in an iterative process. We used Cohen's kappa to validate our analysis, reaching an average of 0.95 for the electricity version and 0.94 for the magnetism counterpart, as has been done in the literature [8].

\section{RESULTS}

We established categories for each question to group our results. We used the two initial states of motion of the charges (at rest or in motion) to separate them to be able to compare results. This way, we can observe the relation that is present in the students' reasoning when engaging with problems with similar surface features.

We categorized students' responses based on the diagrams and the written explanation that they used to answer each problem. In Fig. 2, we present examples of the categories that emerged from the data. We identified four main categories: (i) In the direction of the field, students in this category answered that the force exerted on the charge is parallel to the field (Fig. 2 (a)); (ii) There is no force includes all the answers of students that explicitly stated that the force exerted on the charge is zero (Fig. 2(b)); (iii) Impetus, includes answers of students that state that the direction of the force exerted on the moving charge is a vector sum between the direction of the field and the direction of the initial motion (Fig. 2(c)); and (iv) Perpendicular to the field gathered all the answers in which students used either cross-product or right-hand rule to deduce their answer (Fig. 2 (d)). We decided to label the third category as Impetus, given the consistency with the explanation for this reasoning reported by Hestenes, et al.
[9]. The answers that were not collected in any of these four categories were included under the label Other, which included infrequent answers -or no answer at all- and could not be grouped nor compared between versions $(\sim 3 \%$ or less for the electricity version, and $\sim 4.5 \%$ or less for the magnetism version). Yet, the Impetus and There is no force categories in the magnetism version were kept for comparison purposes.

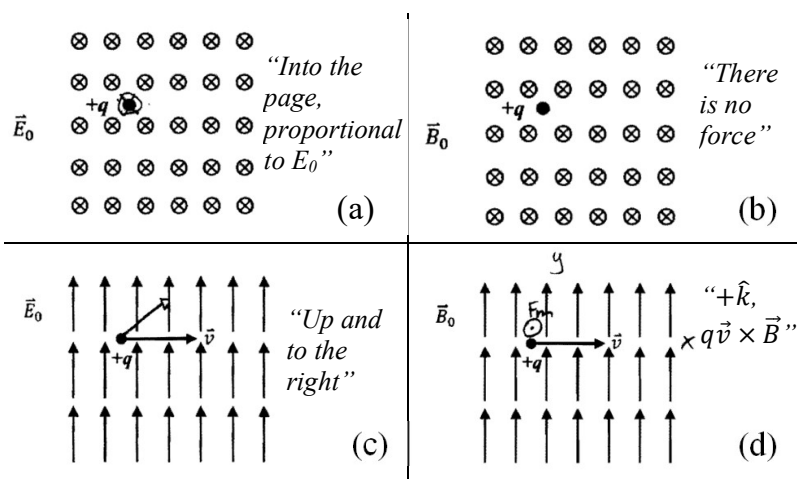

Figure 2. Examples of students' answers used to create categories.

\section{A. Students' conceptions of a charge at rest in a uniform field}

We present in Table I the results of students' answers to the first question, comparing the electricity and the magnetism version. When presented with a charge at rest in a uniform magnetic field, about two-thirds $(65 \%)$ of the students answered correctly, stating that the force exerted on the charge is zero. But, on the electric counterpart, only a much smaller fraction of the students (37\%) correctly deduce that the force exerted on the charge is parallel to the electric field. In this analysis, we describe the most frequent difficulties that students face when answering the electricity question, the magnetism question, and the implications that we can derive from our comparisons and observations.

In the electricity question, the most frequent difficulty found was that there was no force exerted on the charge $(29 \%)$. The most common explanation for this result was "because the charge is not moving." This could imply two different types of reasoning. On the one hand, students might be activating an idea from magnetism to answer an electric field question, concluding that if the charge is at rest, the electric force will be zero (as if it were magnetic force). We think that this might be the case for some students, which implies that the "no-motion, no-force" magnetism idea is quite present in the students' reasoning. On the other hand, students might be activating a well-known difficulty in mechanics, the naïve idea of Impetus. Students might be relating the fact that the charge is not in motion to conclude that there is no force. This is coherent with the results of the charge in motion, which we present in the next section. At first glance, both types of reasoning seem similar, but the conceptions that are used are different. One is the application 
of the magnetic part of the Lorentz force equation, and the other is the notion of Impetus.

The other common difficulty in the electricity question was that the force is perpendicular to the electric field (17\%). Even though the problem does not show the charge in motion, students show a cross-product operation written down, which they have used extensively in magnetism. Since there is no velocity on the charge, the students that used a cross-product operation, would choose perpendicular vectors practically at random. We grouped under this category all perpendicular vectors (namely, up, down, left, and right). The most common explanations of the students grouped in this category included statements of the righthand rule or even the Lorentz magnetic force equation $\vec{F}=$ $q \vec{v} \times \vec{B}$. Considering the difficulty exposed earlier, it seems that students activate different ideas from magnetism (Lorentz force, cross product, and right-hand rule) when answering an electric field question.

In the magnetism question, the most common difficulty was that the force is perpendicular to the field (13\%). It is worthwhile to highlight that the question explicitly stated that the charge was at rest, and there was nothing that could be mistaken for a velocity vector in the diagram. This means that students did not have enough tools from the diagram to make a cross-product between the velocity and the magnetic field. Nevertheless, some students answered that the force exerted on the charge was perpendicular to the field, attempting a cross-product operation. The next-to-most common difficulty was that the magnetic field exerts a force on the charge -in the direction of the field (9\%). This could mean that students use the electric field concept when answering a magnetism question.

When comparing both questions, it is evident that some concepts from magnetism are activated when answering an electric field question, which interfere with the understanding of the concept of the electric field. This is consistent with Scaife and Heckler's [3] results, which will be further addressed in the discussion. There is interference in the other direction, too. Some ideas from electricity used when answering a magnetism question, but the frequency of this occurrence is significantly smaller. This could be a consequence of the magnetism concepts being taught closer to the administration of the test than the electric concepts, as the authors in [3] point out. Students find the concepts from magnetism more readily available -which would also explain why there is a slight interference of electricity concepts in magnetism questions. We can observe that the magnetism concepts are more present and more used by students, regardless of the type of field (electric or magnetic).

\section{B. Students' conceptions of a charge in motion in a uniform field}

The results of the scenario with a charge in motion in a uniform field emphasize the relation between electricity and magnetism from the students' point of view (Table II). In the magnetism question, $69 \%$ of students correctly asserted that the force is perpendicular to the field, while in the electricity question only $16 \%$ of students correctly identified that the electric force is in the direction of the field.

TABLE I. Results of a charge at rest

\begin{tabular}{lcc}
\hline \hline \multicolumn{1}{c}{ Category } & Electricity & Magnetism \\
\hline In direction of the field & $37 \%$ (correct) & $9 \%$ \\
There is no force & $29 \%$ & $65 \%$ (correct) \\
Perpendicular to the field & $17 \%$ & $13 \%$ \\
Other & $17 \%$ & $13 \%$ \\
Total & $100 \%$ & $100 \%$ \\
\hline \hline
\end{tabular}

TABLE II. Results of a charge in motion

\begin{tabular}{lcc}
\hline \hline \multicolumn{1}{c}{ Category } & Electricity & Magnetism \\
\hline In direction of the field & $16 \%$ (correct) & $8 \%$ \\
There is no force & $11 \%$ & $1 \%$ \\
Perpendicular to the field & $41 \%$ & $69 \%$ (correct) \\
Impetus & $16 \%$ & $0 \%$ \\
Other & $16 \%$ & $22 \%$ \\
Total & $100 \%$ & $100 \%$ \\
\hline \hline
\end{tabular}

In this section, we analyze the most common difficulties that students had on each question and our observations when comparing electricity and magnetism.

In the electricity question, $41 \%$ of the students stated that the force exerted on the moving charge was perpendicular to the field, as we present in Table II. This suggests that the surface features of the representation, along with the chargein-motion characteristic of the problem might lead students to think about magnetism, hence showing an incomplete conceptual understanding of the electric field. Students often encounter problems of charges in motion in uniform magnetic fields during instruction, while this is not always (or necessarily) the case when learning about electric fields.

The second most common difficulty in the electricity question was consistent with the Impetus category description. Almost one-sixth of the sample (16\%) indicated with arrows, words, or both that the force exerted on the charge would be parallel to the resulting motion (up and to the right). This data could indicate that students still carry on the well-known misconception of the pre-Galilean 'force' needed to keep objects in motion in mechanics. Furthermore, it seems that students mix this misconception with the superposition principle since they add this Impetus 'force' to the electric force exerted by the electric field.

In the magnetism question, the most common difficulty was that the force is in the direction of the field (8\%). Again, we observe how some students still use the electric field concept as an idea to answer this question. A very low number of students stated that there is no force acting on the charge $(1 \%)$, for which there is no apparent reason since the charge is moving inside the magnetic field. The rest of the students $(22 \%)$ gave answers that could not be inserted in any of the four main categories, with some of them even leaving the question unanswered. This could be an indicator of the complexity of the question and the phenomenon for the students in general. 
When comparing both questions, we can observe the same pattern as in the results of the pair of questions 1 . The interference of the magnetism concepts in the electricity questions is present, but this is not so strong when it goes the other way around, consistent again with the results in [3]. It is also remarkable that the Impetus category was not present in the magnetism version of the test. This could imply that this is not an idea that students think of when answering magnetism questions. However, this might also suggest that there are some similarities observed by students between electricity and mechanics. We will deepen into this idea in the next section.

\section{DISCUSSION AND CONCLUSION}

In this article, we analyze students' answers to two pairs of questions that presented similar surface features between them but with substantial differences such as the type of field or the initial state of motion of the charge. The results obtained through both sets of questions are consistent. For instance, we can observe that a considerable number of students confuse the magnetism concepts in electricity questions ( $46 \%$ for the static-charge scenario; $52 \%$ for the moving-charge scenario), while the counterpart (interferences of electricity concepts in magnetism questions) is significantly lower in both cases $(9 \%$ in the static-charge scenario; $8 \%$ in the moving-charge scenario). This last data supports the idea of a stronger interference of the most recently taught topic (in this case, magnetism) on the concepts of its counterpart (electricity), which is consistent with what was reported by Scaife and Heckler [3]. We remark that, when observing the case of the questions involving a charge in motion, our results delivered very similar information to what was also reported in their work (i.e., in the electricity question $2,41 \%$ of students answered Perpendicular to the field, against $\sim 45 \%$ presented in the literature; or in the magnetism question, $69 \%$ of students correctly answered that the force was perpendicular to the field, against $\sim 70 \%$ reported). An important point of our work is that we obtain evidence that the interference between the concepts of magnetism and electricity is also present in problems where the charge is initially at rest.

Also, the Impetus category arose as an unexpected but remarkable result that we would like to discuss. In [3], there was a report that consistently a certain number of students answered that the force exerted on the charge inside any given field was in the direction of the velocity. The explanation given was that students selected the most explicit direction indicated in the question, but this option was still selected even after instruction. It is interesting that, since in their study, the questions were multiple-choice questions, they did not report any Impetus-like reasoning. Rather, they only identified students that related the force with the initial velocity of the charged particle.
Given the open-ended nature of our study, we could develop from their finding that students not only relate the initial state of motion to the force but that they identify the resulting motion of the particle and answer that the force is in that direction. Following this reasoning, we found in the electricity test a small number of students who answered that there is no force on the particle at rest and answered that the force is in the direction of the motion on the particle with an initial velocity. This is the reason why we think that the concept of Impetus might also be elicited in the case of the particle at rest in the electricity version. Furthermore, we found a small number of students in the electricity test who correctly answered the question in the static-charge case, and then presented an answer that was consistent with the Impetus category in the case of the charge in motion. As we declared before, it appears that a certain number of students, by this point, are unable to get rid of the idea that motion implies force, typical of mechanics courses. When teaching these topics, we recommend making an explicit contrast between at-rest and in-motion cases and differentiating between trajectory and force.

We find interesting that the naïve idea of Impetus is only present when answering electricity questions, and not in the magnetism questions, although in some cases it is unclear if the no-motion answer comes from the Impetus model or from the confusion with the magnetism concept. This could be evidence that students relate the phenomena of electricity to mechanics, while magnetism is treated independently. In the case of magnetism, students are "allowed" to think that if the charge is not in motion, the magnetic field does not exert a force on the charge. Sometimes, students do not understand the meaning of the Lorentz equation in magnetism. Some students might relate the Lorentz force with the idea that motion implies force which, in this case, for a naive reasoning, is true, if the charge is moving, there will be a magnetic force on it. We think that it is important to explain the meaning of the Lorentz force, i.e., that magnetic fields can only exert force on moving charged particles. However, a particle does not need a magnetic force to be in motion.

In this study, we explored the cases of charges at rest and in motion in uniform fields. To have a broader comparison of the understanding of electric and magnetic phenomena, we intend to study other cases, such as the notion of field and superposition principle, and the interactions between charged particles or currents. In future contributions, we aim to continue analyzing and comparing student understanding of electricity and magnetism in more scenarios.

\section{ACKNOWLEDGMENTS}

We thank the Donostia Physics Education Research Group from the University of the Basque Country for providing the tools used for the analysis. We also acknowledge the partial financial support of the School of Engineering and Sciences of Tecnologico de Monterrey. 
[1] D. P. Maloney, T. L. O'Kuma, C. J. Hieggelke, and A. Van Heuvelen, Surveying students' conceptual knowledge of electricity and magnetism, Am. J. Phys. 69, S12 (2001).

[2] Chi, P. Feltovich, and R. Glaser, Categorization and representation of physics problems by experts and novices, Cogn. Sci. 5 (2), 121 (1981)

[3] T. M. Scaife and A. F. Heckler, Interference between electric and magnetic concepts in introductory physics, Phys. Rev. ST Phys. Educ. Res. 7, 010104 (2011).

[4] J. Guisasola, J. M. Almudi, and J. L. Zubimendi, Difficulties in learning the introductory magnetic field theory in the first years of university, Sci. Educ. 88, 443 (2004).
[5] T. M. Scaife and A. F. Heckler, The effect of field representation on student responses to magnetic force questions, AIP Conf. Proc. 951, 180 (2007)

[6] H. Young and R. Freedman, University physics with modern physics (Pearson, Mexico, 2013).

[7] L. C. McDermott and P. Shaffer, Tutorials in Introductory Physics (Pearson Education, Buenos Aires, 2001).

[8] K. Zuza, P. van Kampen, M. De Cock, T. Kelly, and J. Guisasola, Introductory university physics students' understanding of some key characteristics of classical theory of the electromagnetic field, Phys. Rev. Phys. Educ. Res. 14, 020117 (2018)

[9] D. Hestenes, M. Wells, and G. Swackhamer, Force Concept Inventory, Phys. Teach. 30, 141 (1992). 